\title{
ON THE DENSE PACKING OF SPHERES
}

\author{
BY \\ FRED SUPNICK
}

1. Definitions and statement of the theorem. Let

$$
S_{1}, \cdots, S_{n}
$$

be a set of spheres with radii of lengths

$$
r_{1} \leqq r_{2} \leqq \cdots \leqq r_{n}
$$

respectively. Let $S$ be a right circular cylindrical surface with radius of length $r$, such that $r_{n} \leqq r$. A sphere $S_{i}$ will be said to be inside $S$ if its interior is completely in the interior of $S$. Let the spheres (1) be assigned positions inside $S$, no two having interior points in common. We say that the spheres (1) have been packed into $S$, and shall be referred to as a packing. All packings considered in this paper are subject to the restriction:

(A) Let $[p q] \equiv$ distance between the centers of $S_{p}$ and $S_{q}$. Let

$$
S_{i}, S_{j}, S_{k}
$$

be in $S$ with (a) $[i j]=r_{i}+r_{j}$, (b) $[j k]=r_{j}+r_{k}$, (c) $[i k] \geqq r_{i}+r_{k}$. Then the spheres (1) are assumed to be so large that $S_{i}, S_{j}, S_{k}$ cannot be continuously moved in $S$, keeping (a) and (b) true, into a position where (c) is false (cf. §4).

COROLLARY 1. If $x, y$ and $z$ are the respective projections of the centers of the spheres (2) on to the axis of $S$, then $y$ "separates" $x$ and $z$.

CoROLlaRY 2. Let $r_{i}$ and $r_{j}$ be any elements of (1.1), then $r_{i}+r_{j}>r$.

COROLlaRY 3. For any element of (1) $r_{i} \geqq r / 4$.

Let the spheres (1) be packed into $S$. Let $x$ and $y$ denote the projections of the centers of the spheres $S_{i}$ and $S_{j}$ of the packing on to $l$, the axis of $S$. We say that $S_{i}$ and $S_{j}$ are adjacent if the projection on to $l$ of no other center of the spheres in the packing separates $x$ and $y$. Let $D$ denote the smallest right circular frustrum of $S$ and its interior, which encloses the packing. The density of the packing is here defined as the volume ratio of the spheres to $D$.

A packing will be called incompressible if any two adjacent spheres are (1) tangent to each other and (2) each tangent to $S$ at diametrically opposite elements of $S$.

COROLLARY. The centers of the spheres forming an incompressible packing all lie in one axial plane of $S$.

Presented to the Society, August 23, 1946; received by the editors October 20, 1947. 
Let a packing $P$ of the spheres (1) into $S$ be given. Let $l$ be oriented. Then $P$ will be denoted by an ordered array of marks $S_{i_{1}}, \cdots, S_{i_{n}}$ written from left to right so that (1) adjacent marks denote adjacent spheres, and (2) if $x$ and $y$ are the projections of the centers of $S_{i j}$ and $S_{i_{k}}$, where $j<k$, then the orientation of the directed line segment $x y \overrightarrow{ }$ is positive. It is obvious that the density of a packing $S_{i_{1}}, \cdots, S_{i_{n}}$ and its reverse $S_{i_{n}}, \cdots, S_{i_{1}}$ are the same.

We prove the following theorem.

Theorem. Consider the set of all possible packings of the spheres (1) into S, subject to the restriction (A).

(1) The density is greatest for the incompressible packing

$$
\left\{\cdots S_{2 i} \cdots S_{6} S_{4} S_{2} S_{1} S_{3} S_{5} S_{7} \cdots S_{2 i+1} \cdots\right\} \text {. }
$$

(2) The least dense incompressible packing is

$$
\left\{S_{1} S_{n} S_{3} S_{n-2} S_{5} S_{n-4} \cdots S_{n-5} S_{6} S_{n-3} S_{4} S_{n-1} S_{2}\right\} \text {. }
$$

It is clear that if some of the spheres (1) have the same radius, then there are other packings whose densities are equal to those of (3) or (4). If there are no spheres in (1) with equal radii, then it will be clear from the proof that no other packing can have a density equal to that of (3) or (4) (except their reverses).

We note that without the restriction (A) we obtain one of the unsolved packing problems: How can $n$ spheres be packed into a container in the densest fashion? The problem remains unsolved even for the case where the container is a cube or cylinder, and the spheres are equal.

2. Some inequalities. We prove the following lemma.

Lemma 1. Let $a, b, c, d, r$ be positive constants such that $a+b, b+c, c+d$, $d+a$ are each greater than $r$. If $a<c$ and $b<d$, then

$$
(a+b-r)^{1 / 2}+(c+d-r)^{1 / 2}<(b+c-r)^{1 / 2}+(a+d-r)^{1 / 2} .
$$

REMARK. It is obvious that if $a=c$, or $b=d$, then the inequality (5) becomes an equality.

Proof. Let $K$ and $L$ be real constants with $K<L$. Let $x>L$. Then, $(x-K)^{1 / 2}>(x-L)^{1 / 2}$. Let $f(x) \equiv(x-K)^{1 / 2}-(x-L)^{1 / 2}$. Then $f^{\prime}(x)<0$. Thus $f(x)$ is a monotonically decreasing function (in the strong sense) in $L<x<\infty$. Thus if $x_{2}>x_{1}>L$

$$
\left(x_{2}-K\right)^{1 / 2}-\left(x_{2}-L\right)^{1 / 2}<\left(x_{1}-K\right)^{1 / 2}-\left(x_{1}-L\right)^{1 / 2} .
$$

Now let $K=r-c$ and $L=r-a$. Since $a<c$, then $K<L$. Let $x_{2}=d$ and $x_{1}=b$. Since $b<d$, then $x_{1}<x_{2}$. Substituting into (6) we obtain 
(7) $(d-(r-c))^{1 / 2}-(d-(r-a))^{1 / 2}<(b-(r-c))^{1 / 2}-(b-(r-a))^{1 / 2}$.

Transposing the second term of each side, we obtain (5).

Lemma 2. Let $a, b, c, r$ be positive constants such that $a+b$ and $a+c$ are each greater than $r$. If $b<c \leqq r$ and $a \leqq r$ then

$$
c+2(r(a+b-r))^{1 / 2}<b+2(r(a+c-r))^{1 / 2} .
$$

REMARK. If $b=c$; then (8) becomes an equality.

Proof. Since $c-b>0$ and $b$ and $c$ are positive, $c+b>2(b c)^{1 / 2}$. Thus $2 c+b$ $>c+2(b c)^{1 / 2}$, and $2 c-2(b c)^{1 / 2}>c-b$. Therefore

$$
c^{1 / 2}-b^{1 / 2}=\left(2 c-2(b c)^{1 / 2}\right) / 2 c^{1 / 2}>(c-b) / 2 c^{1 / 2} \geqq(c-b) / 2 r^{1 / 2} .
$$

If $a=r$ then (9) implies (8). Suppose $a<r$. In the proof of Lemma 1 we saw that if $K<L, f(x) \equiv(x-K)^{1 / 2}-(x-L)^{1 / 2}$ is a monotonically decreasing function (in the strong sense) for $L<x<\infty$. In (6) let $K=0, L=r-a, x_{1}=b$, $x_{2}=c$. Transposing, we obtain

$$
(a+c-r)^{1 / 2}-(a+b-r)^{1 / 2}>c^{1 / 2}-b^{1 / 2} .
$$

Combining (9) and (10) we obtain

$$
(a+c-r)^{1 / 2}-(a+b-r)^{1 / 2}>(c-b) / 2 r^{1 / 2} .
$$

Multiplying both sides by $2 r^{1 / 2}$ and transposing, we obtain (8).

Lemma 3. Let $K$ be a positive constant. If

$$
\begin{gathered}
0<x \leqq K, \quad 0<y \leqq K, \quad 0<z \leqq K, \\
x+y>K, \quad x+z>K,
\end{gathered}
$$

then

(12.1) $f(x, y, z) \equiv(K(y+x-K))^{1 / 2}+(K(x+z-K))^{1 / 2}-(y z)^{1 / 2}$

is a monotonically increasing function, that is, if $\left(x_{1}, y_{1}, z_{1}\right)$ and $\left(x_{2}, y_{2}, z_{2}\right)$ are in the region (12) and if $x_{1} \leqq x_{2}, y_{1} \leqq y_{2}, z_{1} \leqq z_{2}$, then

$$
f\left(x_{1}, y_{1}, z_{1}\right) \leqq f\left(x_{2}, y_{2}, z_{2}\right) \text {. }
$$

Proof. We note that if

$$
x_{1} \leqq x \leqq x_{2}, \quad y_{1} \leqq y \leqq y_{2}, \quad \text { and } \quad z_{1} \leqq z \leqq z_{2},
$$

then $(x, y, z)$ is also in the region (12).

By the theorem of the mean for functions of three variables

$$
\begin{aligned}
f\left(x_{2}, y_{2}, z_{2}\right)= & f\left(x_{1}, y_{1}, z_{1}\right)+h \cdot f_{x}\left(x_{1}+\theta h, y_{1}+\theta k, z_{1}+\theta l\right) \\
& +k \cdot f_{y}\left(x_{1}+\theta h, y_{1}+\theta k, z_{1}+\theta l\right) \\
& +l \cdot f_{z}\left(x_{1}+\theta h, y_{1}+\theta k, z_{1}+\theta l\right)
\end{aligned}
$$


where $0<\theta<1$ and $h=x_{2}-x_{1}, k=y_{2}-y_{1}, l=z_{2}-z_{1}$. But

$$
\begin{aligned}
f_{x}\left(x_{1}+\theta h, y_{1}+\theta k, z_{1}+\theta l\right) \equiv & \left(K^{1 / 2} / 2\right) \cdot\left(\left(x_{1}+y_{1}+\theta(h+k)-K\right)^{-1 / 2}\right. \\
& \left.+\left(x_{1}+y_{1}+\theta(h+l)-K\right)^{-1 / 2}\right)>0 .
\end{aligned}
$$

Also,

$$
\begin{aligned}
f_{y}\left(x_{1}+\theta h, y_{1}+\theta k, z_{1}+\theta l\right) \equiv & 2^{-1}\left(K^{1 / 2}\left(x_{1}+y_{1}+\theta(h+k)-K\right)^{-1 / 2}\right. \\
& \left.-\left(z_{1}+\theta l\right)^{1 / 2} \cdot\left(y_{1}+\theta k\right)^{-1 / 2}\right) \geqq 0
\end{aligned}
$$

since $x_{1}+\theta h<K$ and $z_{1}+\theta l<K$. Similarly,

$$
f_{z}\left(x_{1}+\theta h, y_{1}+\theta k, z_{1}+\theta l\right) \geqq 0 .
$$

Thus (13) is true.

3. A minimal property of incompressible packings. Let two spheres $A$ and $B$ with centers $a$ and $b$, and radii of lengths $\alpha$ and $\beta$ respectively, be packed into a cylinder $S$. Let $l$ be the axis of $S$, and $r$ the length of the radius. Suppose also that $\alpha+\beta>r$. Let $x$ and $y$ be the projections of $a$ and $b$ respectively upon $l$. We prove the following lemma.

LEMMA 4. The distance between $x$ and $y$ is a minimum if and only if the packing $A, B$ is incompressible.

Proof. If $A$ and $B$ are not mutually tangent, then it is obvious that by translating each parallel to $l$ and toward each other, that $d(x, y)$ (the distance between $x$ and $y$ ) is decreased. Thus let us suppose that $A$ and $B$ are mutually tangent. Suppose one of the spheres, say $B$, is not tangent to $S$. We show how $A$ and $B$ can be rearranged to make $d(x, y)$ smaller, and $B$ tangent to $S$. Let the ray emanating from $a$, which is parallel to $x y \overrightarrow{ }$ and similarly oriented, intersect the sphere $D$ with $a$ as center and radius $(\alpha+\beta)$ in the point $p$. If $p \equiv b$, let $G$ be any great circle on $D$ through $p$. If $p \not \equiv b$, let $G$ be the great circle on $D$ through $b$ and $p$. Let $S^{\prime}$ be the right circular cylinder with $l$ as axis and radius $(r-\beta)$. Since $\alpha+\beta>r, D$ intersects $S^{\prime}$. Starting from $p$, let us traverse $G$ going toward $b$ along the minor arc $p b$. Let $w_{1}$ be the first intersection point with $S^{\prime}$, and $w$ its projection upon $l$. Since $B$ is not tangent to $S$, $w$ falls between $x$ and $y$. Thus, if $B$ is moved so that its center falls on $w_{1}$, then the distance between the projections of the centers of $A$ and $B$ upon $l$ is reduced, that is, it becomes $d(x, y)-d(w, y)$.

Let us now suppose that $A$ and $B$ are each tangent to $S$. Let $S$ be taken with $l$ on the $x$-axis, with $a$ on the positive half of the $y$-axis at the point $(0, r-\alpha, 0)$, and $b$ at the point $(x, y, z)$ with $x>0$. Then the equation of $D$ is

$$
x^{2}+(y-(r-\alpha))^{2}+z^{2}=(\alpha+\beta)^{2}
$$

and the equation of $S^{\prime}$ is

$$
y^{2}+z^{2}=(r-\beta)^{2}
$$


Eliminating $y$ between (15) and (16), we see that $(d x / d z)=0$ if and only $z=0$, with the minimum value of $x$ being

$$
2(r(\alpha+\beta-r))^{1 / 2} \text {. }
$$

Thus, that point of the curve defined by (15) and (16) which is closest to the $y z$-plane is

$$
\left(2(r(\alpha+\beta-r))^{1 / 2}, \beta-r, 0\right) .
$$

This means that if the sphere $B$ is put so that its center falls on the point (18), $A$ and $B$ will be mutually tangent, and each tangent to $S$ at diametrically opposite elements. Thus when the packing is incompressible, the projection on to $l$ of the line segment joining $a$ to $b$ is a minimum, and is given by (17).

If by translations parallel to, and rotations about, the axis of $S$, one packing can be carried into (that is, becomes identical to) another, then we say that the packings are equivalent. It is obvious that the densities of two equivalent packings are identical.

Two packings of the spheres (1) into $S$

$$
S_{i_{1}}, \cdots, S_{i_{n}} \text { and } S_{j_{1}}, \cdots, S_{j_{n}}
$$

will be said to be similarly ordered if (1) $S_{i_{k}} \equiv S_{j_{k}}$ and (2) if the projections of the centers of $S_{i_{1}}, S_{i_{n}}, S_{j_{1}}, S_{j_{n}}$ on to the axis $l$, are $a, b, c, d$ respectively, then the orientation of $a b^{\rightarrow}$ is the same as that of $c d \overrightarrow{\text {. }}$.

"Similar ordering" is an equivalence relation. Let $\{S\}$ denote the set of all packings of the spheres (1) into $S$. Then $\{S\}$ can be subdivided into maximal sets of similarly ordered packings:

$$
\{S\} \equiv\{S\}_{1}+\cdots+\{S\}_{n !}
$$

where any two elements of $\{S\}_{i}$ have similar orderings, and any two elements from distinct sets do not. It is clear that in any $\{S\}_{i}$ there is one and only one class of equivalent incompressible packings.

COROLlaRy. Let $\{S\}_{i}$ be any one of the maximal sets of similarly ordered packings of (19). Then the density of any element of $\{S\}_{i}$ which is not incompressible is less than that of the incompressible packing (that is, any element of the class of equivalent incompressible packings).

Proof. Let

$$
S_{i_{1}}, \cdots, S_{i_{n}}
$$

be an element of $\{S\}_{i}$ which is not incompressible. Let $p_{1}, \cdots, p_{n}$ denote the projections of the respective centers of $(20)$ on to the axis of $S$. Then by Lemma 4 , if the subpacking $S_{i_{j}} S_{i_{j+1}}$ is not incompressible

$$
d\left(p_{j}, p_{i+1}\right)>2\left(r\left(r_{i_{j}}+r_{i_{j+1}}-r\right)\right)^{1 / 2} \text {. }
$$


Since (20) is not incompressible, there must be at least one pair of adjacent spheres which do not form an incompressible subpacking. Thus

$$
r_{1}+\left(\sum_{j=1}^{n-1} d\left(p_{j}, p_{j+1}\right)\right)+r_{n}>r_{1}+\left(\sum_{j=1}^{n-1} 2\left(r\left(r_{i_{j}}+r_{i_{j+1}}-r\right)\right)^{1 / 2}\right)+r_{n}
$$

from which the corollary follows.

We note that if a packing of the spheres (1) into $S$ is densest, then it must be incompressible.

4. The assumption expressed analytically. We prove that the inequality

$$
\left(r\left(r_{1}+r_{2}-r\right)\right)^{1 / 2}+\left(r\left(r_{1}+r_{3}-r\right)\right)^{1 / 2} \geqq\left(r_{2} r_{3}\right)^{1 / 2}
$$

is equivalent to the assumption (A).

Proof. The assumption states that for any relative positions of the packing (2) satisfying the hypothesis of (A), the conclusion holds. Then certainly it holds for those arrangements where the centers of $S_{i}$ and $S_{k}$ are closest. Suppose that the spheres (2) form an incompressible packing in the given order (that is, the projection of the center of $S_{j}$ on to $l$ falls between the projections of the other two centers upon $l$ ). Then if the section of this packing with the plane on which the centers lie is considered, it is seen that the assumption implies that

$$
\left(r\left(r_{i}+r_{j}-r\right)\right)^{1 / 2}+\left(r\left(r_{j}+r_{k}-r\right)\right)^{1 / 2} \geqq\left(r_{i} r_{k}\right)^{1 / 2}
$$

for distinct $i, j$ and $k$. Conversely, if (22) is true, and if the packing (2) is incompressible, then $S_{i}$ and $S_{k}$ cannot have any interior point in common. But for any other similarly ordered packing of the spheres (2), the centers of the end elements cannot be closer than for the incompressible packing. Thus (22) implies the assumption (A).

We now note that if $f(x, y, z)$ is the function defined by (12.1), and if $r_{b} \leqq \min \left(r_{a}, r_{c}\right)$, then

$$
f\left(r_{b}, r_{a}, r_{c}\right) \leqq f\left(r_{a}, r_{b}, r_{c}\right)
$$

since $\left(r\left(r_{b}+r_{c}-r\right)\right)^{1 / 2} \leqq\left(r\left(r_{a}+r_{c}-r\right)\right)^{1 / 2}$ and $\left(r_{a} r_{c}\right)^{1 / 2} \geqq\left(r_{b} r_{c}\right)^{1 / 2}$. Also,

$$
f\left(r_{b}, r_{a}, r_{c}\right) \leqq f\left(r_{c}, r_{a}, r_{b}\right) \text {. }
$$

Thus, since $r_{1} \leqq r_{2} \leqq r_{8}$,

$$
f\left(r_{1}, r_{2}, r_{3}\right) \leqq \min \left(f\left(r_{2}, r_{1}, r_{3}\right), f\left(r_{3}, r_{2}, r_{1}\right)\right) .
$$

Now, let $r_{i}, r_{j}$ and $r_{k}$ be distinct elements of (1.1). By Lemma 3, and bearing in mind that $f(x, y, z)=f(x, z, y)$, we have

$$
\begin{array}{ll}
f\left(\boldsymbol{r}_{i}, \boldsymbol{r}_{j}, \boldsymbol{r}_{k}\right) \geqq f\left(\boldsymbol{r}_{1}, \boldsymbol{r}_{2}, \boldsymbol{r}_{3}\right) & \text { if } i<j<k \text { or } i<k<j, \\
f\left(\boldsymbol{r}_{\boldsymbol{i}}, \boldsymbol{r}_{j}, \boldsymbol{r}_{k}\right) \geqq f\left(\boldsymbol{r}_{2}, \boldsymbol{r}_{3}, \boldsymbol{r}_{1}\right) & \text { if } i<i<k \text { or } k<i<j,
\end{array}
$$

and 


$$
f\left(\boldsymbol{r}_{i}, \boldsymbol{r}_{i}, \boldsymbol{r}_{k}\right) \geqq f\left(\boldsymbol{r}_{3}, \boldsymbol{r}_{1}, \boldsymbol{r}_{2}\right) \quad \text { if } k<j<i \text { or } j<k<i .
$$

Thus by (25)

$$
f\left(\boldsymbol{r}_{i}, \boldsymbol{r}_{j}, \boldsymbol{r}_{k}\right) \geqq f\left(\boldsymbol{r}_{1}, \boldsymbol{r}_{2}, \boldsymbol{r}_{3}\right) .
$$

But if (22) is true for all distinct $i, j, k$, then certainly (21) is true. Conversely if (21) is true, by (26), (22) is true for all $i, j$, and $k$. Thus the assumption (A) is equivalent to (21).

We note that (21) tells us explicitly how large the spheres must be taken.

5. Proof of part (1) of the theorem. By the corollary of $\$ 3$, it becomes necessary to consider only the incompressible packings. Henceforth the term "packing" will mean "incompressible packing." Let $P_{1}$ be any packing of the spheres (1) into $S$. We shall prove part (1) by constructing an ordered set of packings

$$
P_{1}, \cdots, P_{n}
$$

such that

(a) $P_{i}$ is obtained from $P_{i-1}$ by a rearrangement of the spheres of $P_{i-1}$ $(i=2, \cdots, n)$,

(b) $P_{n}$ is the packing (3), and

(c) if $d_{i}$ is the density of $P_{i}(i=1, \cdots, n)$, then

$$
d_{1} \leqq d_{2} \leqq \cdots \leqq d_{n} .
$$

The packing $P$, will be denoted by

$$
S_{1}^{\prime}, \cdots, S_{n}^{\prime} \text {. }
$$

The symbol

$$
\left(\nu ; i_{1}, \cdots, i_{n}\right)
$$

will denote the packing

$$
S_{i_{1}}^{\nu}, S_{i_{2}}^{\nu}, \cdots, S_{i_{n}}^{\nu} \text {. }
$$

$r_{i_{j}}^{\nu}$ will denote the length of the radius of $S_{i_{j}}^{\nu}$. Notice that the superscript of $r$ will be used as an identification mark, and should not be taken as its power.

Let $P_{1}$ be a packing $S_{i_{1}}, \cdots, S_{i_{n}}$ of the spheres (1) which we denote by

$$
S_{1}^{1}, \cdots, S_{n}^{1}
$$

that is, $S_{j}^{1} \equiv S_{i_{j}}$, for the sake of a uniform notation. Let $S_{\theta_{1}}^{1} \equiv S_{1}$ and $S_{k_{1}}^{1} \equiv S_{2}$ ( $S_{i}$ without the superscript denotes the $i$ th sphere from the left in (1)). If $k_{1}>g_{1}$, then we can reverse the packing. If $k_{1}=g_{1}-1$ then we can take $P_{2} \equiv P_{1}$.

(1) $1<k_{1} \leqq g_{1}-2$. We show that the density of 
(30) $\left(1 ; 1,2, \cdots, k_{1}-1, g_{1}-1, g_{1}-2, \cdots, k_{1}+1, k_{1}, g_{1}, g_{1}+1, \cdots, n\right)$ is greater than or equal to that of (29). For, all the $r_{i}(i=1, \cdots, n)$ are positive, and $r_{i}+r_{j}>r$. But $r_{\theta_{1}}^{1} \equiv r_{1}$ and $r_{k_{1}}^{1} \equiv r_{2}$. Therefore by (1.1) $r_{g_{1}}^{1} \leqq r_{k_{1}-1}^{1}$ and $r_{k_{1}}^{1} \leqq r_{g_{1}-1}^{1}$. By Lemma 1

$$
\begin{aligned}
\left(r_{g_{1}}^{1}+r_{k_{1}}^{1}-r\right)^{1 / 2}+\left(r_{k_{1}-1}^{1}\right. & \left.+r_{g_{1}-1}^{1}-r\right)^{1 / 2} \\
& \leqq\left(r_{k_{1}-1}^{1}+r_{k_{1}}^{1}-r\right)^{1 / 2}+\left(r_{g_{1}-1}^{1}+r_{o_{1}}^{1}-r\right)^{1 / 2}
\end{aligned}
$$

Thus, .

$$
\begin{aligned}
r_{1}^{1} & +\left(\sum_{j=1}^{n-1} 2\left(r\left(r_{j}^{1}+r_{j+1}^{1}-r\right)\right)^{1 / 2}\right)+r_{n}^{1} \\
\geqq r_{1}^{1} & +\left(\sum_{j=1}^{k_{1}-2} 2\left(r\left(r_{j}^{1}+r_{j+1}^{1}-r\right)\right)^{1 / 2}\right) \\
& +2\left(r\left(r_{k_{1}-1}^{1}+r_{g_{1}-1}^{1}-r\right)\right)^{1 / 2}+\left(\sum_{j=k_{1}}^{g_{1}-2} 2\left(r\left(r_{j}^{1}+r_{j+1}^{1}-r\right)\right)^{1 / 2}\right) \\
& +2\left(r\left(r_{k_{1}}^{1}+r_{g_{1}}^{1}-r\right)\right)^{1 / 2}+\left(\sum_{j=g_{1}}^{n-1} 2\left(r\left(r_{j}^{1}+r_{j+1}^{1}-r\right)\right)^{1 / 2}\right)+r_{n}^{1}
\end{aligned}
$$

Therefore the density of (30) is greater then or equal to that of (29).

(2) $k_{1}=1$. The density of

$$
\left(1 ; g_{1}-1, g_{1}-2, \cdots, 1, g_{1}, g_{1}+1, \cdots:, n\right)
$$

is greater than or equal to that of (29). $r_{\theta_{1}}^{1}=r_{1}$ and $r_{1}^{1}=r_{2}$. Therefore $r_{1}^{1} \leqq r_{\theta_{1}-1}^{1}$. Since the other conditions of Lemma 2 are also satisfied,

$$
r_{g_{1}-1}^{1}+2\left(r\left(r_{1}^{1}+r_{g_{1}}^{1}-r\right)\right)^{1 / 2} \leqq r_{1}^{1}+2\left(r\left(r_{g_{1}-1}^{1}+r_{g_{1}}^{1}-r\right)\right)^{1 / 2} .
$$

Thus the left side of (32) is greater than or equal to

$$
\begin{aligned}
r_{g_{1}-1}^{1} & +\left(\sum_{j=1}^{g_{1}-2} 2\left(r\left(r_{j}^{1}+r_{j+1}^{1}-r\right)\right)^{1 / 2}\right)+2\left(r\left(r_{1}^{1}+r_{g_{1}}^{1}-r\right)\right)^{1 / 2} \\
& +\left(\sum_{j=g_{1}}^{n-1} 2\left(r\left(r_{j}^{1}+r_{j+1}^{1}-r\right)\right)^{1 / 2}\right)+r_{n}^{1}
\end{aligned}
$$

Therefore the density of (33) is greater than or equal to that of (29).

Now let $P_{2}$ be the packing (30) or (33) according as $k_{1}$ is greater than or equal to 1 , and denote it by

$$
S_{1}^{2}, \cdots, S_{n}^{2}
$$

that is, $S_{j}^{2}$ is identical to the $j$ th element from the left in either (30) or (33). Let $S_{g_{2}}^{2} \equiv S_{1}, S_{\vartheta_{2}-1}^{2} \equiv S_{2}$ and $S_{k_{2}}^{2} \equiv S_{3}$. If $k=g_{2}+1$, then we can take $P_{3} \equiv P_{2}$. 
(1) $g_{2}+1<k_{2}<n$. Then the density of

(37) $\left(2 ; 1,2, \cdots, g_{2}, k_{2}, k_{2}-1, \cdots, g_{2}+2, g_{2}+1, k_{2}+1, k_{2}+2, \cdots, n\right)$ is greater than or equal to that of $P_{2}$. For by Lemma 1

$$
\begin{aligned}
\left(r_{o_{2}}^{2}+r_{k_{2}}^{2}-r\right)^{1 / 2}+\left(r_{g_{2}+1}^{2}+r_{k_{2}+1}^{2}\right. & -r)^{1 / 2} \\
& \leqq\left(r_{g_{2}}^{2}+r_{g_{2}+1}^{2}-r\right)^{1 / 2}+\left(r_{k_{2}}^{2}+r_{k_{2}+1}^{2}-r\right)^{1 / 2}
\end{aligned}
$$

since $r_{g_{2}}^{2} \leqq r_{k_{2}+1}^{2}$ and $r_{k_{2}}^{2} \leqq r_{o_{2}+1}^{2}$

(2) $k_{2}=n$. Then the density of

$$
\left(2 ; 1,2, \cdots, g_{2}, n, n-1, \cdots, g_{2}+1\right)
$$

is greater than or equal to that of $P_{2}$. For by Lemma 2

$$
r_{g_{2}+1}^{2}+2\left(r\left(r_{\theta_{2}}^{2}+r_{n}^{2}-r\right)\right)^{1 / 2} \leqq r_{n}^{2}+2\left(r\left(r_{g_{2}}^{2}+r_{g_{2}+1}^{2}-r\right)\right)^{1 / 2}
$$

since $r_{n}^{2} \leqq r_{g_{2}+1}^{2}$.

(3) $1 \leqq k_{2}<g_{2}-2$. Then by Lemma 1 or Lemma 2 , the density of (39) $\left(2 ; 1,2, \cdots, k_{2}-1, g_{2}-2, g_{2}-3, \cdots, k_{2}+1, k_{2}, g_{2}-1, g_{2}, \cdots, n\right)$ is greater than or equal to that of $P_{2}$, since $r_{g_{2}-1}^{2} \leqq r_{k_{2}-1}^{2}$ and $r_{k_{2}}^{2} \leqq r_{g_{2}-2}^{2}$. By Lemma 1 or Lemma 2, the density of

(40) $\left(2 ; 1,2, \cdots, k_{2}-1, g_{2}-2, g_{2}-3, \cdots, k_{2}+1, k_{2}, g_{2}, g_{2}-1, g_{2}+1, \cdots, n\right)$ is greater than or equal to that of (39), since $r_{\theta_{2}}^{2} \leqq r_{o_{2}-1}^{2}$ and $r_{k_{2}}^{2} \leqq r_{O_{2}+1}^{2}$. Finally, the density of the reverse of (40)

$$
\begin{aligned}
\left(2 ; n, n-1, \cdots, g_{2}+1, g_{2}-1, g_{2}, k_{2}, k_{2}+1, \cdots,\right. \\
\\
\left.g_{2}-3, g_{2}-2, k_{2}-1, k_{2}-2, \cdots, 1\right)
\end{aligned}
$$

is the same as that of $(40)$.

We consider the general case, that is, we show how to construct $P_{v+1}$ from $P_{\boldsymbol{v}}$.

Case I. $\nu=2 m+1$. Let

$$
P_{n}: S_{1}^{2 m+1}, \cdots, S_{n}^{2 m+1}
$$

be such that

(43) $S_{o_{\nu}+i}^{\nu} \equiv S_{2 i+1}(i=0,1, \cdots, m)$, and $S_{o_{\nu}-i}^{\nu^{\prime}} \equiv S_{2 i}(i=1,2, \cdots, m)$.

Let $S_{k_{\nu}}^{2 m+1} \equiv S_{2 m+2}$. If $k_{\nu}=g_{\nu}-m-1$, then let $P_{\nu+1} \equiv P_{\nu}$. of

(1) Suppose $1 \leqq k_{\nu} \leqq g_{\nu}-m-2$. Then by Lemma 1 or Lemma 2 the density 


$$
\begin{aligned}
\left(2 m+1 ; 1,2, \cdots, k_{\nu}-1, g_{\nu}-m-1, g_{\nu}-m-2, \cdots,\right. \\
\left.k_{\nu}+1, k_{\nu}, g_{\nu}-m, g_{\nu}-m+1, \cdots, n\right)
\end{aligned}
$$

is greater than or equal to that of $P_{\nu}$, since $r_{k_{\nu}-1}^{\nu} \geqq r_{o \nu-m}^{\nu}$ and $r_{o \nu-m-1}^{\nu} \geqq r_{k_{\nu}}^{\nu}$.

(2) Suppose $g_{\nu}+m+2 \leqq k_{\nu} \leqq n$. Then by Lemma 1 or Lemma 2 the density of

$$
\begin{aligned}
\left(2 m+1 ; 1,2, \cdots, g_{\nu}\right. & +m, k_{\nu}, k_{\nu}-1, \cdots, \\
& \left.g_{\nu}+m+2, g_{\nu}+m+1, k_{\nu}+1, k_{\nu}+2, \cdots, n\right)
\end{aligned}
$$

is greater than or equal to that of $P_{r}$; also the density of

$$
\begin{array}{r}
\left(2 m+1 ; 1,2, \cdots, g_{\nu}-m-1, g_{\nu}+m, g_{\nu}+m-1, \cdots, g_{\nu}-m+1,\right. \\
g_{\nu}-m, k_{\nu}, k_{\nu}-1, \cdots, g_{\nu}+m+2, g_{\nu}+m+1, \\
\left.k_{\nu}+1, k_{\nu}+2, \cdots, n\right)
\end{array}
$$

is greater than or equal to that of (45). Finally the density of the reverse of (46)

$$
\begin{array}{r}
\left(2 m+1 ; n, n-1, \cdots, k_{\nu}+2, k_{\nu}+1, g_{\nu}+m+1, g_{\nu}+m+2, \cdots\right. \\
k_{\nu}-1, k_{\nu}, g_{\nu}-m, g_{\nu}-m+1, \cdots, g_{\nu}+m-1, \\
\left.g_{\nu}+m, g_{\nu}-m-1, g_{\nu}-m-2, \cdots, 1\right)
\end{array}
$$

is equal to the density of (46).

Case II. $\nu=2 m$. Let

$$
P_{v}: S_{1}^{2 m}, \cdots, S_{n}^{2 m}
$$

be such that

$$
\begin{array}{rr}
S_{\sigma_{\nu}+i}^{\nu} \equiv S_{2 i+1} & (i=0,1,2, \cdots, m-1) \text { and } \\
S_{\sigma_{\nu}-i}^{\nu} \equiv S_{2 i} & (i=1, \cdots, m) .
\end{array}
$$

Let $S_{k_{v}}^{2 m} \equiv S_{2 m+1}$. If $k_{v} \equiv g_{\nu}+m$, then let $P_{v+1} \equiv P_{\nu}$. of

(1) Suppose $g_{\nu}+m+1 \leqq k_{\nu} \leqq n$. Then by Lemma 1 or Lemma 2 the density

$$
\begin{aligned}
\left(2 m ; 1,2, \cdots, g_{\nu}+m-1, k_{\nu}, k_{\nu}-1, \cdots, g_{\nu}+m+1,\right. \\
\\
\left.g_{\nu}+m, k_{\nu}+1, k_{\nu}+2, \cdots, n\right)
\end{aligned}
$$

is greater than or equal to that of $P_{\nu}$ since $r_{g_{\nu}+m-1}^{2 m} \leqq r_{v_{\nu}+1}^{2 m}$ and $r_{k_{y}}^{2 m} \leqq r_{g_{\nu}+m}^{2 m}$. of

(2) Suppose $1 \leqq k_{\nu} \leqq g_{\nu}-m-2$. Then by Lemma 1 or Lemma 2 the density

$$
\begin{array}{r}
\left(2 m ; 1,2, \cdots, k_{\nu}-1, g_{\nu}-m-1, g_{\nu}-m-2, \cdots, k_{\nu}+1,\right. \\
\left.k_{\nu}, g_{\nu}-m, g_{\nu}-m+1, \cdots, n\right)
\end{array}
$$


is greater than or equal to that of $P_{v}$; and the density of

$$
\begin{aligned}
& \left(2 m ; 1,2, \cdots, k_{\nu}-1, g_{\nu}-m-1, g_{\nu}-m-2, \cdots, k_{\nu}+1, k_{\nu},\right. \\
& \left.g_{\nu}+m-1, g_{\nu}+m-2, \cdots, g_{\nu}-m, g_{\nu}+m, g_{\nu}+m+1, \cdots, n\right)
\end{aligned}
$$

is greater than or equal to that of (51). Finally, the density of the reverse of (52)

$$
\begin{array}{r}
\left(2 m ; n, n-1, \cdots, g_{\nu}+m+1, g_{\nu}+m, g_{\nu}-m, g_{\nu}-m+1, \cdots,\right. \\
g_{\nu}+m-2, g_{\nu}+m-1, k_{\nu}, k_{\nu}+1, \cdots, g_{\nu}-m-2, \\
\left.g_{\nu}-m-1, k_{\nu}-1, k_{\nu}-2, \cdots, 1\right)
\end{array}
$$

is equal to the density of (52).

Thus, depending on $\nu$ and $k_{\nu}, P_{\nu+1}$ is taken as (44), (47), (50) or (53). $P_{2}$ is obtained from $P_{1}, P_{3}$ from $P_{2}, \cdots, P_{n}$ from $P_{n-1}$. Thus we obtain an ordered set (27) satisfying the properties (a), (b) and (c) stated at the beginning of $\$ 5$.

This completes the proof of part (1) of the theorem. It is clear from the statements of Lemmas 1 and 2 that the density of the packing (3) is greater than the density of any other packing (besides the reverse of (3)), if no two $r$ 's of (1.1) are equal. $d_{n}$ is actually greater than $d_{1}$ in (27), if the strong inequalities hold at least once in the repeated application of Lemmas 1 and 2.

6. Proof of part (2) of the theorem. We shall prove part (2) of the theorem by constructing an ordered set of packings

$$
Q_{0}, \cdots, Q_{n}
$$

such that (a) $Q_{i}$ is obtained from $Q_{i-1}$ by a rearrangement of the spheres of $Q_{i-1}(i=1, \cdots, n)$, (b) $Q_{n}$ is the packing (4), and (c) if $d_{i}$ is the density of $Q_{i}(i=0,1, \cdots, n)$, then

$$
d_{0} \geqq d_{1} \geqq d_{2} \geqq \cdots \geqq d_{n} .
$$

The packing $Q_{p}$ will here also be denoted by (28); and (28.1) will denote (28.2). Let $Q_{0}$ be a packing $S_{i_{1}}, \cdots, S_{i_{n}}$ of the spheres (1). Writing $S_{j}^{0}$ for $S_{i_{j}}$ $(j=1, \cdots, n), Q_{0}$ becomes $S_{1}^{0}, \cdots, S_{n}^{0}$.

(1) If $S_{1}^{0} \equiv S_{1}$, then let $Q_{1} \equiv Q_{0}$. If $S_{n}^{0} \equiv S_{1}$ then we can take $Q_{1}$ identical to the reverse of $Q_{0}$. Let $S_{g_{0}}^{0} \equiv S_{1}$. Suppose $1<g_{0}<n$. Then the density of

$$
\left(0 ; g_{0}, g_{0}-1, \cdots, 1, g_{0}+1, g_{0}+2, \cdots, n\right)
$$

is less than or equal to that of $Q_{0}$. For by Lemma 2

$$
r_{g_{0}}^{0}+2\left(r\left(r_{1}^{0}+r_{g_{0}+1}^{0}-r\right)\right)^{1 / 2} \geqq r_{1}^{0}+2\left(r\left(r_{g_{0}}^{0}+r_{g_{0}+1}^{0}-r\right)\right)^{1 / 2}
$$

since $r_{g_{0}}^{0} \leqq r_{1}^{0}$.

(2) Let $Q_{1}: S_{1}^{1}, \cdots, S_{n}^{1}$, be the packing (55). If $S_{n}^{1} \equiv S_{2}$, let $Q_{2} \equiv Q_{1}$. Let $S_{\theta_{1}}^{1} \equiv S_{2}$. Suppose $1<g_{1}<n$. Then by Lemma 2 , the density of 


$$
\left(1 ; 1,2, \cdots, g_{1}-1, n, n-1, \cdots, g_{1}\right)
$$

is less than or equal to that of $Q_{1}$ since $r_{\theta_{1}}^{1} \leqq r_{n}^{1}$. Let $Q_{2}: S_{1}^{2}, \cdots, S_{n}^{2}$ be (57).

We now consider the general case, that is, we show how to obtain $Q_{v+1}$ from $Q_{\nu}: S_{1}^{\nu}, \cdots, S_{n}^{\nu}(\nu \geqq 3)$. Let

$$
f(i) \equiv i \sin ^{2}(i \pi / 2)+(n-i+2) \cos ^{2}(i \pi / 2)
$$

and

$$
g(i) \equiv(i+1) \sin ^{2}(i \pi / 2)+(n-i+1) \cos ^{2}(i \pi / 2) .
$$

Case I. $\nu=2 m$.

(1) $m=2 m_{1}$. Let

$$
S_{i}^{\nu} \equiv S_{f(i)}(i=1, \cdots, m) \quad \text { and } \quad S_{n-i+1}^{\nu} \equiv S_{g(i)}(i=1, \cdots, m) .
$$

Let $S_{k_{\nu}}^{4 m_{1}} \equiv S_{f(m+1)}$. If $k_{\nu}=m+1$ then let $Q_{\nu+1} \equiv Q_{\nu}$. Suppose $m+2 \leqq k_{\nu} \leqq n-m$. Then by Lemma 1, the density of

$$
\left(4 m_{1} ; 1,2, \cdots, m, k_{\nu}, k_{\nu}-1, \cdots, m+1, k_{\nu}+1, k_{\nu}+2, \cdots, n\right)
$$

is less than or equal to that of $Q_{4 m_{1}}$ since $r_{m}^{4 m_{1}} \geqq r_{k_{\nu}+1}^{4 m_{1}}$ and $r_{m+1}^{4 m_{1}} \geqq r_{k_{\nu}}^{4 m_{1}}$.

(2) $m=2 m_{1}+1$. Let

$$
S_{i}^{\nu} \equiv S_{f(i)}(i=1, \cdots, m) \quad \text { and } \quad S_{n-i+1}^{\nu} \equiv S_{g(i)}(i=1, \cdots, m) .
$$

Let $S_{k_{\nu}}^{4 m_{1}+2} \equiv S_{f(m+1)}$. If $k_{\nu}=m+1$ then let $Q_{\nu+1} \equiv Q_{\nu}$. Suppose $m+2 \leqq k_{\nu} \leqq n-m$. Then by Lemma 1, the density of

(60) $\left(4 m_{1}+2 ; 1,2, \cdots, m, k_{\nu}, k_{\nu}-1, \cdots, m+1, k_{\nu}+1, k_{\nu}+2, \cdots, n\right)$

is less than or equal to that of $Q_{4 m_{1}+2}$ since $r_{m}^{4 m_{1}+2} \leqq r_{k_{v}+1}^{4 m_{1}+2}$ and $r_{m+1}^{4 m_{1}+2} \leqq r_{k_{v}}^{4 m_{1}+2}$.

Case II. $\nu$ odd.

(1) $\nu=2 m+1, m=2 m_{1}$. Let

(61) $\quad S_{i}^{\nu} \equiv S_{f(i)}(i=1, \cdots, m+1)$ and $S_{n-i+1}^{\nu} \equiv S_{g(i)}(i=1, \cdots, m)$.

Let $S_{k_{\nu}}^{2 m+1} \equiv S_{g(m+1)}$. If $k_{\nu}=n-m$, let $Q_{\nu+1} \equiv Q_{\nu}$. Suppose $m+2 \leqq k_{\nu}<n-m$. Then by Lemma 1 , the density of

$$
\begin{aligned}
\left(2 m+1 ; 1,2, \cdots, k_{\nu}-1, n-m, n-m-1, \cdots,\right. \\
\left.k_{\nu}, n-m+1, n-m+2, \cdots, n\right)
\end{aligned}
$$

is less than or equal to that of $Q_{4 m_{1}+1}$ since $r_{k_{\nu}-1}^{4 m_{1}+1} \leqq r_{n-m+1}^{4 m_{1}+1}$ and $r_{k_{\nu}}^{4 m_{1}+1} \leqq r_{n-m}^{4 m_{1}+1}$.

(2) $\nu=2 m-1, m=2 m_{1}$. Let

$$
S_{i}^{\nu} \equiv S_{f(i)}(i=1, \cdots, m) \quad \text { and } S_{n-i+1}^{\nu} \equiv S_{o(i)}(i=1, \cdots, m-1) .
$$

Let $S_{\boldsymbol{k}_{\nu}}^{2 m-1} \equiv S_{g(m)}$. If $k_{\nu}=n-m+1$, let $Q_{v+1} \equiv Q_{\nu}$. Suppose $m+1 \leqq k_{\nu} \leqq n-m$. Then by Lemma 1 , the density of 


$$
\begin{aligned}
\left(4 m_{1}-1 ; 1,2, \cdots, k_{\nu}-1, n-m\right. & +1, n-m, \cdots, \\
k_{v} & n-m+2, n-m+3, \cdots, n)
\end{aligned}
$$

is less than or equal to that of $Q_{4 m_{1}-1}$ since $r_{k_{\nu}-1}^{4 m_{2}-1} \geqq r_{n-m+2}^{4 m_{1}-1}$ and $r_{k_{y}}^{4 m_{y}-1} \geqq r_{n-m+1}^{4 m_{p}-1}$.

Thus $Q_{v+1}$ is taken as (59), (60), (62) or (63) according as $\nu$ is of the form $4 m_{1}, 4 m_{1}+2,4 m_{1}+1$, or $4 m_{1}-1$. $Q_{3}$ is obtained from $Q_{2}, Q_{4}$ from $Q_{3}, \cdots, Q_{n}$ from $Q_{n-1}$. Thus we obtain an ordered set (54), satisfying the properties stated at the beginning of $\S 6$.

This completes the proof of part (2) of the theorem. It is clear from the statements of Lemmas 1 and 2 that the density of (4) is less than the density of any other (incompressible) packing (besides the reverse of (4)), if no two $r$ 's of (1.1) are equal. $d_{n}$ is actually less than $d_{1}$ in any set (54) if the strong inequalities hold at least once in the repeated application of Lemmas 1 and 2.

Columbia University, NEW YORK, N. Y. 